\title{
CORRELATION BETWEEN VISUAL FUNCTIONS AND OPTICAL COHERENCE TOMOGRAPHY, FUNDUS AUTO FLUORESCENCE AND FUNDUS FLUORESCEIN ANGIOGRAPHY FINDINGS IN TREATMENT-NAIVE DIABETIC MACULAR EDEMA
}

\author{
Sara Samy Gamal Eldin, Mohamed Omar Rashed, Sherif Nabil Embabi, Maged \\ Maher Roshdy, Ahmed Mohamed Habib
}

Department of Ophthalmology Faculty of Medicine-Ain Shams University, Cairo Egypt

Corresponds:

Sara Samy Gamal Eldin

E.male:

ophthafundus88@yahoo.com Tel.: 01006271541

Received: 7/3/2019

Accepted: 2/4/2019

\begin{abstract}
:
Background: Diabetic macular edema (DME) is a sightthreatening consequence of diabetic retinopathy. So, identifying reliable methods for DME prognosis is actually very helpful. Multimodal retinal imaging tools provide us with these predictive prognostic biomarkers
\end{abstract}

Aim of the Work: To correlate between visual functions (visual acuity and color vision) with macular features of OCT, FAF and FFA in patients with untreated (treatment naive) DME as a guide for visual prognosis of these patients.

Patients and Methods: Fifty eyes of 35 diabetic patients with untreated clinically significant macular edema (CSME)underwent best corrected visual acuity (BCVA) determination (logMAR), fluoresce in angiography(FFA;FAZ size, macular leakage pattern, areas of capillary dropout)optical coherence tomography (OCT; central point foveal thickness [CPFT], volume, outer and inner retinal layers integrity[ONL,INL],hyper-reflective foci[HRF], subfoveal choroidal thickness[SFCT]); fundus auto fluorescence (Hyper FAF; absent or increased (FAF, single or multiple spots). Linear correlation and three-way analysis of covariance were used for statistics.

Results: In OCT, we found that CPFT \& ORL integrity is significantly well correlated to visual function (BCVA, color vision). However, CSFT was only correlated to visual acuity but not color vision. Regarding FFA parameters, especially (FAZ size, areas of capillary drop out), we found that these parameters are correlated significantly to visual acuity but not color vision. On the other hand, we found that the presence of hyper FAF spots was not related significantly to visual acuity but related to a significant level to color vision. However, a number of spots were not correlated to visual acuity or color vision. There was significant correlation between retinal thickness in OCT and type of leakage in FFA. Also, large cystoid spaces and FAF spots in the fovea were significantly correlated together.

Conclusions: Integration between different retinal imaging tools in DME helps to find alternative and less invasive way in diagnosis.FAF is new, simple non-invasive imaging mode to assess $R P E$ function with evolving application in DME.

Keywords: Diabetic Macular Edema, Best Corrected Visual Acuity, Optical Coherence Tomography, Fundus Auto Fluorescence, Fundus Fluoresce in Angiography. 


\section{INTRODUCTION:}

Diabetic macular edema (DME) is a major cause of vision loss in diabetic patients. The prevalence of DME among diabetic patients ranges from $1 \%$ to $5.7 \%$, while its prevalence in patients with diabetic retinopathy (DR) is from $2.7 \%$ to $11 \%{ }^{(1)}$.Most important risk factors for DME are the type of diabetes (type I or II), the treatment method (insulin, oral hypoglycemic drugs, or diet only), and the mean duration of diabetes ${ }^{(2)}$.The functional impact of DME is usually determined by measuring best corrected visual acuity (BCVA). However, BCVA does not depend only on macular thickness. For a given degree of diabetic maculopathy, a wide range of visual acuity may be observed. Therefore, changes in retinal thickness have low predictive value in relation with BCVA changes $^{(1)}$.The gold standard for diagnosing DME fundus was slit lamp biomicroscopy. Nevertheless, we usually need optical coherence tomography (OCT) for early detection of DME and monitoring treatment response $^{(3)}$. Fluoresce in angiography (FA), which is an invasive modality, is an important tool for diagnosis and characterization on of DME disease ${ }^{(4)}$.DME related visual affection negatively impacts patients' visual functioning and overall quality of life ${ }^{(5)}$.

\section{Imaging Modalities}

Multimodal imaging and full assessment in cases of Diabetic Macular Edema (DME) is mandatory in order to complete the whole disease picture and fulfilling the whole data about effect of diabetes on the retina. ${ }^{(6)}$

\section{Fundus auto-fluorescence:}

FAF had been used in evaluation in cases of macular dystrophy and age-related maculopathy. However, its role in DME is not fully understood ${ }^{(7)} \mathrm{AF}$ have a role in judging the visual potential and prognosis of patients, with long standing DME, by assessing the integrity and function of the underlying (Retinal Pigment Epithelium) RPE and in turn the health of the adjacent photoreceptors $^{(8)}$.FAF is thought to derive from (LF) lipofuscin (fluorophores in the human retina) in RPE cells, reflecting some aspect of RPE function and integrity. Fluorophores are chemical structures that possess fluorescent properties when exposed to light of an appropriate wavelength ${ }^{(9)}$.LF and its $\mathrm{AF}$ are low at the fovea, increase gradually to a maximum at $7-13^{\circ}$ from the fovea, and then decrease toward the periphery. From the foveal center, significant increase in the signal is seen at the margin of the fovea, followed by a gradual increase toward the outer macula ${ }^{(9)}$. The AF intensity of fundus is characterized by a very low intensity. As a result, camera systems with high sensitivity and/or imageaveraging capabilities with alignment to account for eye movements are required to record the FAF with acceptable signal/noise ratios. Confocal scanning laser ophthalmoscopy (cSLO) optimally addressed these requirements ${ }^{(10)}$. Fundus camera systems are actually more affected by scattering than are cSLO system. These factors may contribute to a reduced ability to discern smaller retinal vessels as compared with the cSLO. The wavelengths used in the fundus camera are not absorbed by macular pigment or fluorescein $^{(9)}$. Abnormalities in the FAF image is very much dependent on the quality of the recorded image. Many factors can influence image quality as crystalline lens opacities and aging ${ }^{(11)}$.

FAF showed $81 \%$ sensitivity and $69 \%$ specificity in detecting cystoid macular edema, offering a rapid and noninvasive technique in DME. AF help in demarcation of areas of the cysts with CME, it is unlikely to replace the role of $\mathrm{OCT}^{(3)}$. Auto fluorescence (AF) may have a role in laser retreatment of DME, particularly with subthreshold laser where burns may not be clinically detectable yet easily apparent with autofluorescence ${ }^{(8)}$. 


\section{Fundus fluorescein angiography}

Fundus fluorescein angiography (FFA) is the gold standard for diagnosing various retinal and choroidal abnormalities ${ }^{(12)}$. Fundus fluorescein angiography (FFA) provides a lot of information in DR and DME. It can determine the size and regularity of foveal avascular zone (FAZ), number and location of micro aneurysms. Also, it can detect intra retinal microvascular abnormalities (IRMA) and early neovascularization and areas of capillary non perfusion $^{(12)}$.

Fluorescein angiography can accurately localize leakage source and its intensity. According to the leakage pattern in FA, DME has been classified into two patterns: focal and diffuse Eyes with DME can demonstrate either leakage pattern, or more commonly, a mixture of both. Clinically speaking, it is not always simple to differentiate focal from diffuse edema and indeed several mixed forms do exist ${ }^{(13)}$. The severity of leakage on FA is correlated both with the severity of outer retinal thickening and cystic changes of the inner and outer retina. Capillary non-perfusion on FA is associated with loss and disruption of the inner retinal layers and (DIRL) on $\mathrm{OCT}^{(14)}$.

\section{Optical coherence tomography:}

Regarding DME, OCT assesses retinal changes both qualitatively (i.e., pattern of DME, presence and character of cysts, integrity, fluid localization, and reflectivity of the individual retinal layers) and quantitatively (i.e., sectorial retinal thickness, macular volume, and central retinal thickness [CRT]). It is therefore essential for the diagnosis, characterization and ultimately for the follow up of DME. ${ }^{(15)}$ In DME, apart from maculopathy analysis, OCT provides precious information on other retinal structures such as the vitreoretinal interface, retinal nerve fiber layers (RNFL), ganglion cell complex (GCC), as well as the choroid. ${ }^{(16)}$ In addition to DME,
OCT allows the identification of several other lesions occurring in the setting of nonproliferative (i.e., hard exudates, MAs, Hyper-reflective spots (HRS), pseudocysts, cotton-wool spots) and proliferative (i.e., neovascularization $[\mathrm{NV}]$, tractional retinal detachment, vitreoschisis, hemorrhage $)^{(17)}$. The orientation of photoreceptor layer status at the fovea has been categorized, including inner segment ellipsoid zone (EZ), continuity of the external limiting membrane (ELM) band and the presence of hyperreflective foci (HRF) in the outer retinal layers ${ }^{(18)}$. A correlation with visual acuity was documented. Eyes with an intact inner segment EZ or ELM band had significantly better visual acuity than eyes with a discontinuous or absent inner segment EZ or ELM band. ${ }^{(19)}$ In few patients, visually significant ischemic maculopathy may also occur in the absence of DME. Macular ischemia is associated with disorganization and loss of inner retinal layers, as well as reductions in retinal sensitivity ${ }^{(20)}$. Microaneurysm turnover has been considered as a prognostic biomarker of the development of DME. Initial studies found that loss of visual acuity is well correlated with foveal thickening, although many involved small sample sizes and lacked best-corrected ETDRS visual acuities. Several studies have also demonstrated that foveal thickness measured by OCT is correlated with $\mathrm{VA}^{(21)}$. On the other hand, other studies revealed that OCT has failed to become a surrogate for evaluation of visual function in DME patients, as it shows just moderate correlation to best corrected visual acuity (BCVA) data ${ }^{(3)}$.

\section{AIM OF THE WORK:}

To investigate the correlation between visual functions (visual acuity and color vision) with macular features of OCT, FAF and FFA in patients with untreated (treatment naive) DME as a guide for visual prognosis of these patients. 


\section{PATIENTS AND METHODS}

This cross-sectional study included thirty-five patients with naïve diabetic macular edema. Patients had been recruited from ophthalmology clinic in Ain Shams University (ASU) Hospital.

Then, they were categorized according to baseline BCVA into 2 groups:

Group 1: patients with baseline BCVA $\log$ MAR $\leq 0.5(\geq 6 / 18)$.

Group 2: patients with baseline BCVA $\log$ MAR $>0.5(<6 / 18)$.

Written consent was taken from all patients in accordance with the tenets of the Helsinki protocol.

\section{Inclusion criteria}

- Type I or II diabetes mellitus patients.

- Patients' age ranging from 18-75 years.

- Patients have center involving DME.

- Patients' BCVA between 3/60 (log $\mathrm{MAR}=2.00)$ and $6 / 9(\log \mathrm{MAR}=0.2)$

\section{Exclusion criteria:}

- Clinically significant media opacities.

- Preexisting glaucoma.

- Any recent intraocular surgery less than 6 months ago.

- Any previous vitreoretinal surgery.

- Any previous treatment modality for DR (Diabetic Retinopathy) or DME (Diabetic Macular Edema).

- Preexisting any other vitreoretinal diseases other than DR.

- High axial myopia (more than 6 diopters).

\section{All eyes underwent the following evaluation procedures:}

1) Meticulous history taking:

The age and gender of the patient were noted as well as any special habits.
Medical history was obtained extensively including the type, duration and treatment received for diabetes and whether the patient was controlled on the given therapy. History about any other medical conditions such as hypertension, renal diseases were asked about.

The patients were asked about any previous treatment modalities for diabetic eye retinopathy or maculopathy as laser or IVI. Many patients were excluded in from the study at this point.

Best-corrected visual acuity (BCVA) was obtained in either Snellen or decimal notation prior to being converted into logMAR for the statistical facility.

2) Color vision assessment using Ishihara Pseudo-Isochromatic Test.

The test contains 24 plates for recognition of the normal color vision and red-green color vision defect. In a well-illuminated room with patients wearing their glasses (and reading glasses for presbyopic patients), plates from 1 to 13 were demonstrated to the literate patients. In illiterate patients, plates from 20-24 were demonstrated. In case of the correct answer in the most of plates, normal color vision was reported. Plates from 14-19 were not used to analyze type of colour vision defect as it was out of the scope of our study.

3) Comprehensive ophthalmic examination was done via slit-lamp biomicroscopy.

The Haag-Streit slit lamp was used. Fundus examination was carried out utilizing the 90D lens (Volk, USA). Any significant media opacity (such as corneal opacity or cataract) was noted and led to the further exclusion of more patients. 
4) Intraocular pressure was measured using the Goldmann applanation tonometer (GAT; Haag Streit).

5) Color fundus photography using VX-20 Retinal camera (KOWA, Japan). This was more of an objective method to document the visible fundus findings. Diabetic maculopathy, any new vessels were noted as well as the classical signs of diabetic retinopathy.

6) Spectral-Domain OCT (SD-OCT) was carried out usingRS-3000 device (OCT Retinas can Advance, NIDEK, Japan). The patients initially received a drop of mydriatic agent (Cyclopentolate 1\%) and were examined approximately 30 minutes later to improve imaging capability.

\section{OCT analysis protocol:}

- Macular map including central subfield thickness, single point foveal thickness.

- Single linear scans (commenting on the integrity of all macular layers including photoreceptor layer, photoreceptor outer segment/inner segment (IS/OS) junction, ELM, Inner retinal layers, presence of hyper reflective foci, vitreoretinal interface).

- Subfoveal choroidal thickness.

Three structures were analyzed in detail: the ELM, IS/OS segment junction as well as hyper reflective foci. 360 -degree radial scans centered on the fovea were obtained for all eyes in a clockwise manner.

7) FAF using VX-20 Retinal camera with conventional short-wavelength (blue) excitation light about $488 \mathrm{~nm}$, commenting on the presence of any hyper- auto fluorescence (single or multiple) spots in the macula.
8) FFA using VX-20 Retinal camera commenting on FAZ (size using widest diameter, perifoveal capillary network), the pattern of leakage in the foveal zone (focal or diffuse) and areas of capillary non-perfusion (involving the macular area within two-disc diameters from the fovea).

9) HBA1c was measured in all patients as a guide for glycemic control in the previous three months.

\section{Statistical Methods}

The collected data were coded and statistically analyzed using IBM SPSS statistics (Statistical Package for Social Sciences) software version 23.0, IBM Corp., Chicago, USA) and Medcalc Statistics 18.9.1 (bvba, Belgium).

Descriptive statistics were done for quantitative data as minimum and maximum of the range as well as mean $\pm \mathrm{SD}$ (standard deviation) for quantitative normally distributed data, median and 2.5-97.5 $\mathrm{P}$ for non-normally distributed, while it was done for qualitative data as number and percentage.

Statistical analyses were done for quantitative variables using Chi-Square test was used to examine the relationship between two qualitative variables, MannWhitney $U$ test to examine relation on two groups for non-parametric. The level of significance was taken at $\mathrm{P}$ value $<0.05$ is significant, otherwise is non-significant.

\section{RESULTS:}

The study group included 35 patients, 13 males $(37.2 \%)$ and 22 females (62.8\%). Regarding study group characteristics with specific parameters distribution are shown in table $1,2 \& 3$. 
Table (1):Median \& distribution of patients' age \& HBA1c

\begin{tabular}{|l|c|c|c|}
\hline & Median & $2.5-97.5$ P & P value \\
\hline Age (years) & 53.5 & $24.50-71.2$ & 0.0011 \\
\hline HBA1c \% & 8.8 & $7-14.2$ & 0.0001 \\
\hline
\end{tabular}

Table (2):Mean \& distribution of patients' duration of DM, BCVA

\begin{tabular}{|l|c|c|c|}
\hline & Mean & $95 \%$ CI & P value \\
\hline BCVA (log MAR) & 0.69 & $0.60-0.77$ & 0.205 \\
\hline DM duration (years) & 11.8 & $10.2-13.4$ & 0.624 \\
\hline
\end{tabular}

Table (3):Median \& distribution of patients' CSFT, CPFT \& FAZ size

\begin{tabular}{|l|c|c|c|}
\hline & Median & $2.5-97.5 \mathrm{P}$ & P value \\
\hline FAZ $(\mathrm{mm})$ & 0.64 & $0.40-1.20$ & 0.0001 \\
\hline CPFT $(\mu \mathrm{m})$ & 360 & $229-825$ & 0.0069 \\
\hline CSFT $(\mu \mathrm{m})$ & 383 & $298-791$ & 0.0006 \\
\hline
\end{tabular}

Table (4): Correlation between DME risk factors and BCVA \& CSFT

\begin{tabular}{|c|c|c|}
\hline \multicolumn{3}{|l|}{ DM duration } \\
\hline & Correlation coefficient $\mathrm{r}$ & $\mathrm{P}$ value \\
\hline BCVA (Log MAR) & 0.19 & 0.186 \\
\hline CSFT $(\mu \mathrm{m})$ & -0.065 & 0.653 \\
\hline \multicolumn{3}{|l|}{ HBA1c } \\
\hline & Correlation coefficient $\mathrm{r}$ & $\mathrm{P}$ value \\
\hline BCVA (Log MAR) & 0.212 & 0.140 \\
\hline CSFT $(\mu \mathrm{m})$ & 0.096 & 0.508 \\
\hline
\end{tabular}

As regards the correlation between patients' risk factors and BCVA (table 4), there were weak correlations between BCVA and type, duration of DM and level of HBA1c. Also, the relation between BCVA and the presence of HTN or renal impairment was not clinically significant. On correlating medical patients' data and central macular thickness, there were no direct relationship between CSFT and type, duration of DM, level of HBA1c, and presence of HTN and renal impairment.

Table (5):Correlation bet. BCVA \& specific parameters in OCT, FFA and FAF

\begin{tabular}{|l|l|c|}
\hline & \multicolumn{1}{|c|}{ Correlation coefficient $\mathrm{r}$} & $\mathrm{P}$ value \\
\hline CSFT $(\mu \mathrm{m})$ & 0.459 & $\mathrm{P}=0.0008$ \\
\hline CPFT $(\mu \mathrm{m})$ & 0.5273 & $\mathrm{P}=0.0001$ \\
\hline FAZ size $(\mathrm{mm})$ & 0.3079 & $\mathrm{P}=0.0296$ \\
\hline
\end{tabular}

As Regarding OCT parameters (table 5), specifically (CPFT, CSFT, ORL, INL, HRF), we found that CPFT \& ORL integrity is significantly well correlated to visual function (BCVA, color vision).However, CSFT was only correlated to visual acuity but not color vision. Regarding FFA parameters, especially (FAZ size, areas of capillary drop out), we found that these parameters are correlated significantly to visual acuity but not color vision. On the other hand, we found that the presence of hyper FAF spots was not related significantly to visual acuity but related to a 
significant level to color vision. But a number of spots were not correlated to visual acuity or color vision.

The study group was divided according to their BCVA (Log MAR) into group (1) with BCVA (Log MAR) $<=0.5$ and group (2) with BCVA (Log MAR) $>0.5$. Then, a comparison was done in all previous OCT, FFA \&FAF parameters between the 2 mentioned groups.

In OCT parameters, there was a statistically significant difference between the 2 groups in CSFT, CPFT \& presence of retinal cystoid spaces. However, there were no significant difference in SFCT, outer \&

Table (6):Correlation between CSFT \& SFCT.

\begin{tabular}{|l|c|c|}
\hline \multirow{2}{*}{} & \multicolumn{2}{|c|}{ SFCT } \\
\cline { 2 - 3 } & Correlation coefficient $\mathrm{r}$ & $\mathrm{P}$-value \\
\hline CSFT & -0.1756 & $\mathrm{P}=0.2326$ \\
\hline
\end{tabular}

\section{DISCUSSION:}

The main concern of this study was to search if there were a correlation between visual functions (mainly BCVA and color vision) and some structural parameters detected in different modalities of retinal imaging (OCT, FFA and FAF).

Regarding OCT parameters correlation with baseline best corrected visual acuity, we found that BCVA is correlated with high significance to CSFT, CPFT, presence of cystoid spaces and ONL integrity but there were no linear correlation to HRF, INL integrity and SFCT.

While the study done by Sun et al. that included 120 eyes, showed that worse baseline VA was associated with increased CST, presence of DRIL, the presence of large cysts and less EZ reflectivity. With following up BCVA at 4 and 8 months, only inner retinal layers integrity and presence of HRF between the 2 groups

In FFA parameters, there was no significant difference in FAZ size between the 2 groups, but the presence of areas of capillary drop out was statistically significant related to visual acuity between the 2 groups.

In FAF parameters, the presence of hyper FAF spots and even their multiplicity was not related to different degrees of visual acuity in comparing the 2 groups.

On studying the relation between retinal thickness in OCT and type of leakage in FFA, they were well correlated.

However, there was no correlation between retinal thickness and subfoveal choroidal thickness in OCT (table 6).

Large cystoid spaces and FAF spots in the fovea were significantly correlated together.

DRIL remained a reliable predictor of visual acuity $^{(22)}$.

On comparing our results regarding this part, the difference can be related to their inclusion criteria that did not set a condition for only naïve cases of center involving $\mathrm{DME}$, as all treatment procedures for DME can themselves alter retinal microstructure. Moreover, during the follow up period, patients received IVI of anti-VGEF or macular laser that can be partially responsible for retinal layers changes.

Moreover, a study published in 2006 by Brian et al. showed that increased retinal thickness and presence of CME were significantly correlated with worse visual acuity in 160 eyes with different patterns of DME. ${ }^{(23)}$

A study conducted by Otani and his colleagues in 2010, they found that BCVA is strongly correlated with lesser extent of Ellipsoid Zone and ELM disruption but 


\section{Sara Samy Gamal Eldin, et al.,}

inversely correlated to central macular thickness. ${ }^{(24)}$

In that context, same results were declared from the study done by Anjali and his colleagues in 2010.

In a study by Uji and his colleagues, they compared between the site of HRF in the outer and inner retinal layers. They found that HRF were present more in the inner retina. They also found that BCVA was significantly worse in eyes with HRF in the outer retinal layers than in eyes without them. In our study, we did not specify the exact location of HRF whether in the ORL or IRL. ${ }^{(25)}$

On searching for previous studies about the correlation between baseline BCVA and foveal choroidal thickness in naïve DME cases, we did not find any documented relation.

On correlating relation between the FFA parameters and baseline BCVA, our study revealed a strong association between BCVA and both of FAZ size and presence of areas of capillary dropout.

A study conducted by Sim and his colleagues revealed strong association between the FAZ size and baseline VA in DME in moderate and severe grades of diabetic macular ischemia (DMI) ${ }^{(26)}$.

As regards the correlation between FAF parameters and baseline VA, we searched for presence and number of hyper FAF spots in the macula. We found that VA was related inversely to the presence of hyper FAF spots and a number of hyper FAF spots, but the difference was not statistically significant.

Our results are matching with results of a study done by Chung et al. in 2012. They revealed a directly proportional relation between LogMAR VA with increased FAF in the fovea. However, their patients were evaluated before and after the IVI of Bevacizumab $^{(8)}$. In a study conducted by
Vujosevic et al. (2011), the same correlation was found ${ }^{(3)}$.

In contrast to the study by Yoshitake et al. in 2015, they stated Log MAR VA was correlated negatively with the mean FAF signals in the parafoveal area. ${ }^{(27)}$ Differences between this study and ours are larger study population (103 eyes) in comparison to ours (50 eyes) and using cSLO for FAF rather than fundus camera in our study.

Regarding OCT parameters correlation with color vision, we found that color vision was correlated to CPFT and ONL integrity with high significant difference but correlated to CSFT, HRF and INL integrity with low significance.

While, on correlating FFA parameters with color vision, we stated that increased FAZ size and presence of areas of capillary dropout were related to affected color vision, but difference was not statistically significant.

On assessing correlation between FAF imaging and color vision, presence of multiple hyper FAF spots in the macula was inversely related to color vision with high statistical significance level.

Limitations of our study regarding this part were that we did not analyze the type of color vision defect whether the red or green color and grading of color vision defect was not done. Also, a number of patients with defective color vision were small that, to some extent, limited statistical analysis.

On reviewing the literature, we did not find studies discussing such correlation between retinal multimodal imaging and color vision as a part of visual function.

The secondary concern of this study was to search for correlation between parameters in different retinal imaging modalities. This can help to find new concepts to integrate and complement information from different imaging techniques. This merging assists in 
understanding DME pathology and sequence of events in this disease.

First, we assessed the relation between macular thickness and subfoveal choroidal thickness. There was a weak inverse correlation between them.

Many studies showed comparable results at this point. In a study done in 2014 by Ünsal and his team, they found a statistically moderate negative correlation between central macular and foveal choroidal thickness. ${ }^{(28)}$

However, our results were not matching with a study done by Kim and his colleagues. Their study showed that cases with DME had a thicker subfoveal choroid than cases without $\mathrm{DME}^{(29)}$.

The controversy between their results and ours can be attributed to the difference in the study population. Their study included patients treated by laser photocoagulation not only naïve patients.

Also, while correlating macular thickness in OCT with Type of fluorescein angiographic perifoveal leakage (either focal or diffuse), we found a strong correlation between them with statistically significant difference.

On reviewing literature, many other studies had matching results with ours in this point. For example, a study was done by Yeung and his colleagues (2009). They found that the severity of leakage in FA is well correlated with the degree of edema elicited in both outer and inner retinal layers using $\mathrm{OCT}^{(30)}$.

Lastly, on searching for any association between the presence of hyper FAF spots and cystoid spaces in OCT, we found a strong association between them with a highly statistically significant difference.

Although they used Confocal Scanning Laser Ophthalmoscope, our results were comparable to results declared by Pece and his team (2010). They found a positive correlation between cystic increased FAF and CME with a $P$ value of 0.001 . They divided cases of hyper FAF spots into single, multiple and combination of both. So, they concluded that FAF might be another useful easy test to distinguish CME rapidly and noninvasively ${ }^{(10)}$.

Moreover, many other studies documented this relation as a study conducted by and his team (2011). They stated a positive correlation between macular FAF and not only CME in OCT but also in FFA cystoid pattern ${ }^{(3)}$.

Also, a study done by Yoshitake and his colleagues (2015) showed the same previous association $^{(27)}$.

\section{Conclusion:}

Integration between different retinal imaging tools is great benefit in diagnosis and management of DME.FAF is simple non-invasive imaging mode to assess RPE function with evolving application in DME. In naïve DME, Visual function are well correlated to specific OCT, FFA and FAF parameters. Moreover, cystoid spaces in the macula cause attenuation of FAF signal leading to hypoautofluorescent spot/s.

\section{REFERENCES:}

1. Shen YC, Xu X, Liu K (2014): Fundus auto fluorescence characteristics in patients with diabetic macular edema. Chinese Medical Journal; 127:1423-1428.

2. Muni AM, Kohly RP, Lee EQ, et al. (2013): Prospective study of inflammatory biomarkers and risk of diabetic retinopathy in the diabetic control and complication trial. JAMA Ophthalmol; 131: 514-21.

3. Vujosevic S, Casciano M, Pilotto E, Boccassini B, Varano M, Midena E (2011): Diabetic Macular Edema: Fundus Auto fluorescence and Functional Correlations. Invest Ophthalmol Vis Sci; 52(1): 442-8.

4. Rabbani H, Allingham MJ, Mettu PS, Cousins SW, Farsiu S (2015): Fullyautomatic segmentation of fluorescein 


\section{Sara Samy Gamal Eldin, et al.,}

leakage in subjects with diabetic macular edema. Invest Ophthalmol Vis Sci; 56: 1482-1492

5. Davidov E, Breitscheidel L, Clouth J, et al. (2009): Diabetic retinopathy and healthrelated quality of life. Graefes Arch ClinExpOphthalmol; 247:267-72.

6. Kang SW, Park CY, Ham DI (2004): The Correlation between Fluorescein Angiographic and Optical Coherence Tomographic Features in Clinically Significant Diabetic Macular Edema. Am J Ophthalmol; 137(2): 313-22.

7. Vujosevic S, Trento B, Bottega E (2012): Scanning laser ophthalmoscopy in the retromode in diabetic macular edema. Acta Ophthalmol; 90: e374-e380

8. Chung H, Park B, Shin HJ, Kim HC (2012): Correlation of fundus auto fluorescence with spectral-domain optical coherence tomography and vision in diabetic macular edema. Ophthalmology; 119:1056-65.

9. Dithmar S and Holz FG (2018): Fluorescence Angiography and Fundus Auto Fluorescence in Ophthalmology. In: Fluorescence Angiography in Ophthalmology. Hart WM (ed.), eBoolk Packages, Chapter 9, pp 31-53.

10. Pece A, Isola V, Holz F, Milani P, Brancato R (2010): Auto fluorescence imaging of cystoid macular edema in diabetic retinopathy. Ophthalmologica; 224: 230-5.

11. Holz FG, Spaide RF, Schmitz-Valckenberg S, Bird AC (2007): Auto fluorescence Imaging with the Fundus Camera. In: Atlas of Fundus Auto fluorescence Imaging. Chapter 5, PP 49-53.

12. Rasquinha A, Bappal A, Arunachalam C (2016): Fundus Fluorescein Angiography in Diabetic Retinopathy: Correlation of Angiographic Findings to the Clinical Maculopathy. IOSR Journal of Dental and Medical Sciences (IOSR-JDMS); 15(2): 8088.

13. Horii $\mathrm{T}$, Murakami $\mathrm{T}$, Nishijima K, Akagi T, Uji A, Arakawa N, Muraoka Y, Yoshimura N (2012): Relationship between fluorescein pooling and optical coherence tomographic reflectivity of cystoid spaces in diabetic macular edema. Ophthalmology; 119(5): 1047-55

14. Ho J, Sull AC, Vuong LN, Chen Y Liu J, Fujimoto JG, Schuman JS, Duker JS (2009): Assessment of Artifacts and Reproducibility across Spectral- and TimeDomain Optical Coherence Tomography Devices. Ophthalmology; 116(10): 196070.

15. Meyer CH, Saxena S, Sadda SR (2017): Spectral Domain Optical Coherence Tomography-Based Imaging Biomarkers and Hyper spectral Imaging. In: Spectral Domain Optical Coherence Tomography in Macular Diseases. Chapter 7, pp 105-115

16. Bandello F, Zarbin MA, Lattanzio R, et al. (2017): Management of Diabetic Retinopathy. DevOphthalmol. Basel, Karger; 60: 28-37

17. Tremolada G, Pierro L, de Benedetto U, et al. (2011): Macular micropseudocysts in early stages of diabetic retinopathy. Retina; 31: $1352-1358$

18. Kang SW, Park CY, Ham DI (2004): The Correlation between Fluorescein Angiographic and Optical Coherence Tomographic Features in Clinically Significant Diabetic Macular Edema. Am J Ophthalmol; 137(2): 313-22.

19. Sophie R, Lu N, Campochiaro PA (2015): Predictors of Functional and Anatomic Outcomes in Patients with Diabetic Macular Edema Treated with Ranibizumab. Ophthalmology; 122(7): 1395-401.

20. Keane PA and Sadda SR (2011): Predicting visual outcomes for macular disease using optical coherence tomography. Saudi J Ophthalmol; 25(2):145-58.

21. Massin P, Girach A, Erginay A, Gaudric A (2006): Optical coherence tomography: a key to the future management of patients with diabetic macularoedema. Acta Ophthalmol Scand; 84(4):4 66-74.

22. Sun JK, Lin MM, Lammer J, Prager S, Sarangi R, Silva PS, Aiello LP (2014): Disorganization of the retinal inner layers as a predictor of visual acuity in eyes with center-involved diabetic macular edema. JAMA Ophthalmol; 132(11): 1309-16. 
23. Brian YK, Scott DS, Peter KK (2006): Optical Coherence Tomographic Patterns of Diabetic Macular Edema. American Journal of Ophthalmology. Volume 142, Issue 3, Pages 405-412.

24. Otani T, Yamaguchi Y, Kishi S (2010): Correlation between visual acuity and foveal microstructural changes in diabetic macular edema. Retina, volume 30 - issue 5 - $\mathrm{p}$ 774-780.

25. Uji A, Murakami T, Nishijima K, Akagi T, Horii T, Arakawa N, Muraoka Y, Ellabban AA, Yoshimura N (2012): Association Between Hyper reflective Foci in the Outer Retina, Status of Photoreceptor Layer, and Visual Acuity in Diabetic Macular Edema. American Journal of Ophthalmology, Volume 153, Issue 4, Pages 710-717.

26. Sim DA, Keane PA, Zarranz-Ventura J, Fung S, Powner MB, Platteau E, Bunce CV, Fruttiger M, Patel PJ, Tufail A, Egan CA (2013): The Effects of Macular Ischemia on Visual Acuity in Diabetic Retinopathy.
Investigative Ophthalmology \& Visual Science, 54, 2353-2360.

27. Yoshitake S, Murakami T, Uji A, Unoki N, Dodo Y, Horii T, Yoshimura N (2015): Clinical relevance of quantified fundus auto fluorescence in diabetic macular oedema. Eye (Lond); 29(5): 662-9.

28. Ünsal E, Eltutar K, Zirtiloglu $\mathrm{S}$, et al. (2014): Choroidal thickness in patients with diabetic retinopathy. Clin Ophthalmol; 8: 637-642.

29. Kim JT, Lee DH, Joe SG, Kim JG, Yoon YH (2013): Changes in Choroidal Thickness in Relation to the Severity of Retinopathy and Macular Edema in Type 2 Diabetic Patients. Retina, Investigative Ophthalmology \& Visual Science; 54: 3378-3384.

30. Yeung L, Lima VC, Garcia P, Landa G, Rosen RB (2009): Correlation between spectral domain optical coherence tomography findings and fluorescein angiography patterns in diabetic macular edema. Ophthalmology; 116(6): 1158-67. 


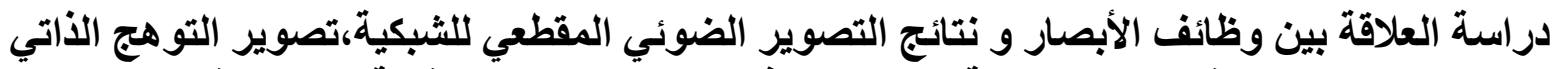

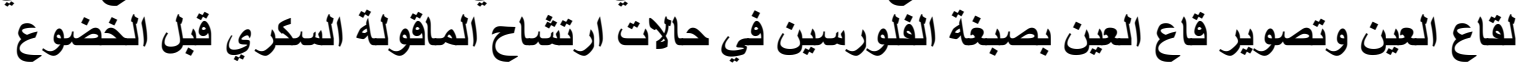
للعلاج

ساره سامى جمال الدين، محمد عمر راثدا، شريف نبيل إمبابي، ماجد ماهر رشدى،

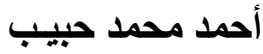

قسم طب وجر احة العيون ـ كلية الطبـ جامعة عبن شمس

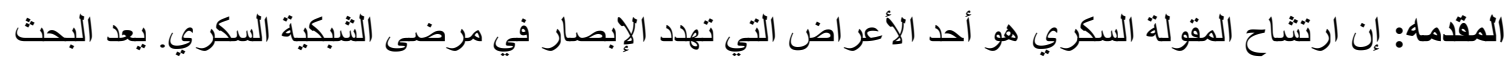

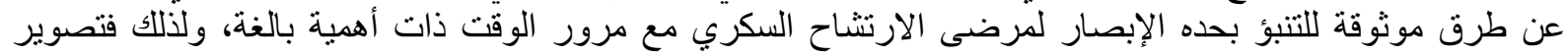

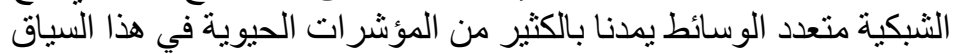

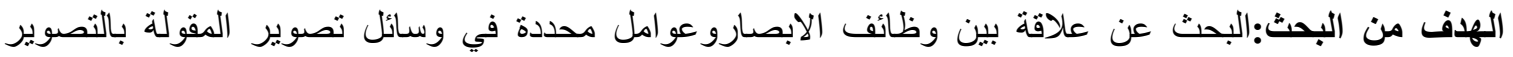

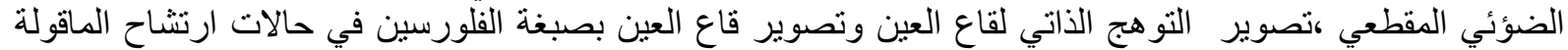

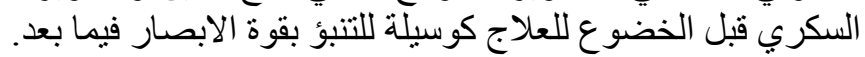

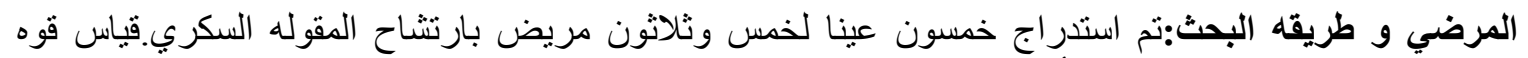

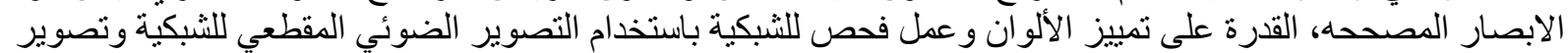

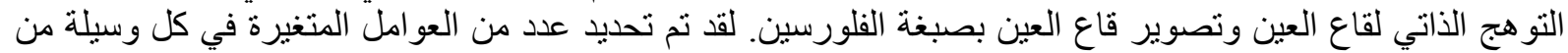

وسائل تصوير الثبكية ومقارنتها بدقه بحده الإبصار لمعرفه درجه الفئه الارتباط بينهما.

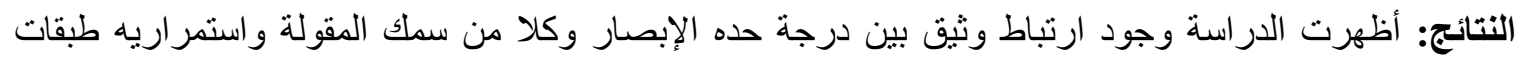

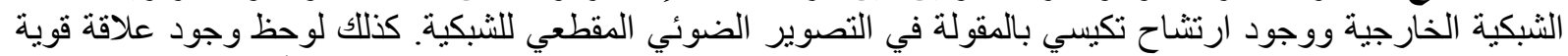

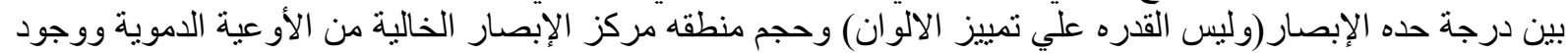

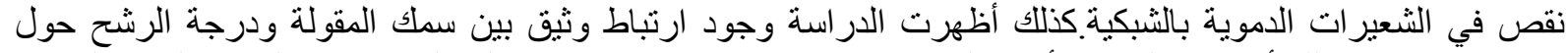

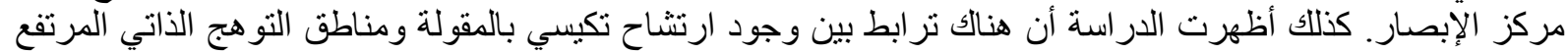

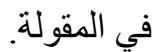

الاستناجات: يوجد علاقات وثثقة بين عوامل مختلفة في مختلف وسائل تصوير الثبكية.يعد تصوير المقولة باستخدام

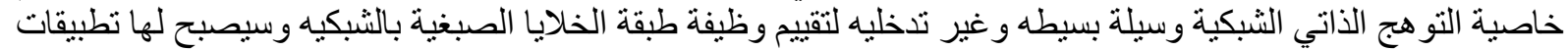
عديدة في حالات ارتثاح المقوله السكري في المستقبل القريب. 